\title{
Pluripotent stem cell technology: a promising remedy for hypopigmentation disorders
}

\begin{abstract}
Pigment cells - epidermal melanocytes play physiological role in providing defense against harmful UV rays. Defect or deficiency of melanocytes and/or melanocyte stem cells can lead to pigmentation disorder such as vitiligo. The vitiligo forms white patches on the skin on the body. It is an autoimmune disease because the pigment inducing cells are damaged. Amongst its therapies, are UV light, cosmetic cover-up and corticosteroid local application. Human epidermis has been produced in vitro from mature epidermal stem cells of donors to provide the cell remedy. Source of pluripotent stem cells, either of embryonic origin or genetic reprogramming offers a substitute for epidermal cell treatment as these cells are immortal and pluripotent - theoretically capable of providing any number of cells of any desired phenotype. Keratinocytes and melanocytes resulting from pluripotent stem cells can be used for pathological modelling of genodermatoses allowing recognition of new diseasespecific pharmacological treatment. We discuss the current approaches and imminent scenario of stem cells in hypopigmentation.
\end{abstract}

Keywords: stem cell, melanocytes, vitiligo, melanin, pigmentation
Volume 2 Issue 5 - 2017

\author{
Kamal Uddin Zaidi,' Sharique AA, ${ }^{2}$ Ayesha \\ SA, ${ }^{2}$ Vijay Thawani' \\ 'Biotechnology Pharmacology Laboratory CSRD, Peoples \\ University, India \\ ${ }^{2}$ Department of Biotechnology and Zoology, Saifia College of \\ Science, India
}

\begin{abstract}
Correspondence: Kamal Uddin Zaidi, Biotechnology Pharmacology Laboratory CSRD, Peoples University, Bhopal, (M.P) 462037, India, Email Zaidi.kamal92@gmail.com
\end{abstract}

Received: April 23, 2017| Published: May 25, 2017
Abbreviations: Escs, epithelial stem cells; Melscs, melanocytes stem cells; Ncscs, neural crest stem cells; Ipscs, patient-specific stem cells; Hfscs, hair follicle stem cells; ORS, outer root sheath; BMP, bone morphogenetic proteins; EDN3, endothelin 34

\section{Introduction}

The epidermal melanocytes protect the skin from UV rays and their functional destruction causes pigmentation disorders. The mutations of melanocyte stem cells cause melanomas. The mechanism of melanocyte differentiation and defining characteristics of melanocyte stem cells in humans are still not fully known. The autologous cultured melanocytes may be useful in the treatment of vitiligo. ${ }^{1,2}$ In contrast to the foreskin melanocytes, expansion of adult melanocytes is not easy. Transplanted pigment cells are known to have repaired the affected area of skin discoloration from vitiligo. Vitiligo affects about two million people in the US. Vitiligo occurs when the body considers melanocytes, cells which give color to the skin, as foreign. In vitiligo the body's own immune system attacks those cells, hence it is an autoimmune disease. Hair follicle has three types of stem cells which are vital to hair development. These incorporate epithelial stem cells (ESCs), melanocytes stem cells (MelSCs) and neural crest stem cells (NCSCs) which are known as human fetal stem cells (hFSCs). ${ }^{3}$ Modeling of neural crest induction, melanocyte specification, and disease-related pigmentation defects in hESCs and patient-specific iPSCs has been reported. ${ }^{4}$ The safety and tolerability of subretinal transplantation of human embryonic-stem-cell (hESCs)-derived retinal pigment epithelium in Asians for the treatment of macular degeneration has been reported. ${ }^{5}$ It has been confirmed that Proteaseactivated receptor- 2 is involved in melanogenesis by mediating stem cell factor production in keratinocytes. ${ }^{6}$ The enthusiasm for vitiligo exploration is coordinated towards the repositories of stem cells, especially the hFSCs. We hereby summarize recent advances in studies of pluripotent stem cells and its utility in vitiligo with specific accentuation on hFSCs.

\section{Reconstruction of pigmentary system using stem cell technology}

Melanin production gives the skin its characteristic pigmentation and increases during sun exposure to protect the cells from the DNAdamaging effects of UV light. Melanocytes have been generated from human embryonic stem cells. The approach involves growing the stem cells in a carefully controlled manner while subjecting them to the specific chemical signals that drive the formation of melanocytes in a developing embryo. It has not been possible to generate melanocytes at different stages of development including mature, fully functional and immature precursors. How melanocytes develop ad function normally, and how failure in these processes lead to diseases, is a matter of study.

The hair follicle is a constantly renewing, where $66 \%$ of the lower follicle (travel part) totally is recovered over the hair cycle, while the staying upper lasting segment is kept up. ${ }^{7}$ The irregular hair cycle comprises three phases of hair follicle viz. development (anagen), trailed by a relapse stage (catagen), and a resting stage (telogen). Melanocytes show up at the onset of anagen stage where they effectively multiply and separate into developed melanocytes. Throughout catagen, the melanocytes are drained from the follicles by apoptosis. Melanocytes go missing in telogen hair follicle until melanogenesis starts in the ensuing anagen stage. Given this regenerative cycle of melanogenesis, the presence of the stem cells for follicular melanocytes has been recommended over 10years. ${ }^{8}$

Pluripotent stem cell technology offers a promising approach for studying human melanocyte development and disease. Timed exposure to activators of WNT, Bone morphogenetic proteins (BMP), and Endothelin 3 (EDN3) signaling triggers the sequential induction of neural crest and melanocyte precursor fates under dual-SMADinhibition condition. ${ }^{4}$ Using a SOX10::GFP human embryonic stem cell (hESC) reporter line, it was demonstrated that the temporal onset of WNT activation is critical for human neural crest induction. 
Subsequent maturation of hESC-derived melanocytes yields a pure population that matches the molecular and functional properties of adult melanocytes. Melanocytes from Hermansky-Pudlak syndrome and Chediak-Higashi syndrome patient specific induced PSCs (iPSCs) reproduced the ultrastructural features of disease-associated pigmentation defects (Figure 1).
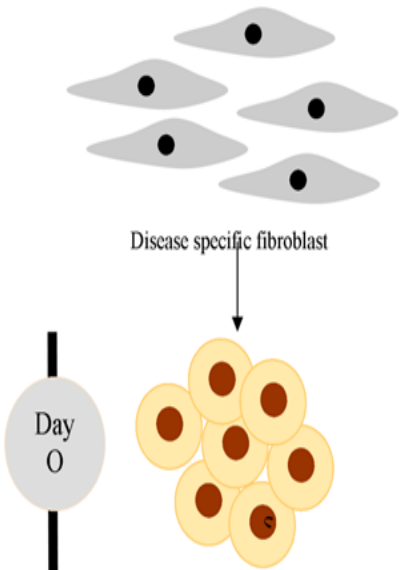

Pluripotent stem cell
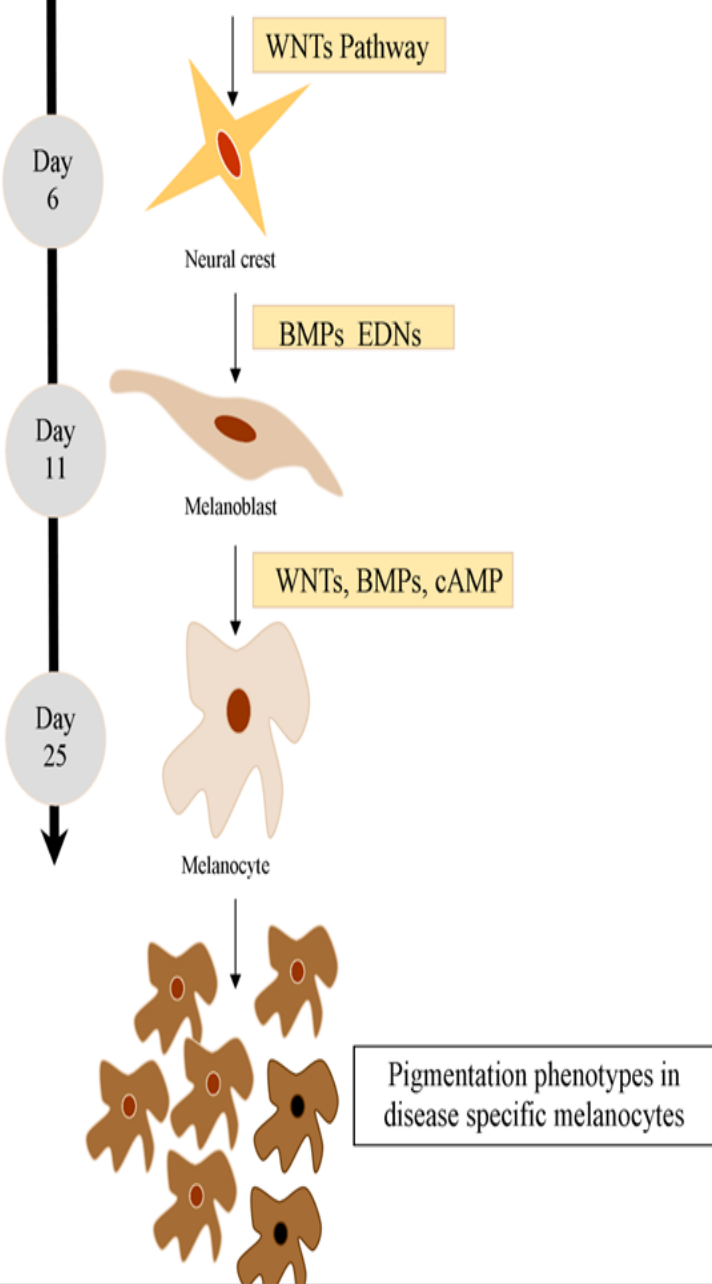

Figure I Disease-specific melanocytes that realistically recapitulate pigmentation defects can be derived from human pluripotent stem cells using a stepwise differentiation pattern.

Skin stem cells reside in the stratum basal of the epidermis, sebaceous glands, and bulge area of the hair follicles. ${ }^{9}$ The HFSCs continuously supply new cells to the bulb during the anagen phase. ${ }^{10}$ The outer root sheath (ORS) of the hair follicle is a rich source of a type of HFSCs called melanocyte stem cells (MelSCs). Hair follicle melanocytes play an important role in repigmentation of vitiliginous lesion. Migration of the precursor melanocytes in the mid portion of the hair follicle, found to be MelSCs have been implicated in the re-pigmentation following both chemical and chemical and physical stimulus. ${ }^{11}$ Common therapeutic modalities such as tacrolimus, phototherapy and dermabrasion act through MelSCs. Newer cellular techniques have explored the use of ORS hair follicle suspension in surgical treatment of vitiligo. Advancement of melanocyte and stem cell research have identified various cytokines, growth factors and regulations involved in proliferation and differentiation of melanoblasts, which can be used for autologous in-situ melanocyte regeneration. ${ }^{12}$

Melanocyte reservoir has been also found in ORS of the white hair in lesional skin. ${ }^{13}$ These MelSCs were proposed to be responsible for repigmentation in vitiligo by separating and migrating upwards along the surface of the hair follicle to nearby epidermis (Figure 2). In transgenic mice HFSCs in the bulge region migrated up into the epidermis to cause peri-follicular repigmentation which later spread in concentric pattern causing diffuse repigmentation. ${ }^{14}$ This occurred in the presence of steel factor (SLF, ligand for kit receptor) expression in the epidermis. These interpretations recommended that SLF provided new routes connecting the follicular ORS and the epidermis, along which the melanoblast could migrate to occupy vacant niches. In vitiliginous lesions, there was the destruction of selectively DOPA-positive melanocytes, whereas the DOPA-negative MelSCs in the outer root sheath (ORS) of the hair follicle were spared. ${ }^{15}$ This reservoir of nonfunctional melanocytes, MelSCs are present in the lesional epidermis of patients with vitiligo, even after disease of 25years duration. ${ }^{16}$

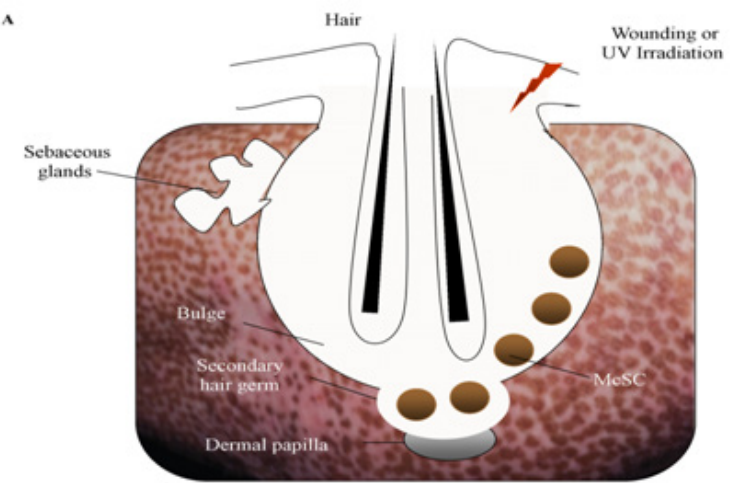

B

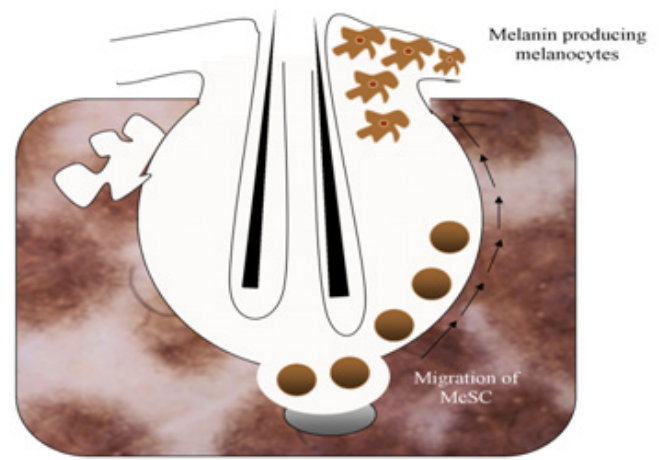

Figure 2 Shows that after loss of epidermal melanocytes by injuring or UV light (a) McSCs from the stem cells area of the proximal hair follicle epithelium move into the recovering epidermis to multiply, hence creating new separated; pigment producing melanocytes (b). 


\section{Conclusion}

Stem cells are the master cells of the human body which can divide to produce their own copies as well as other types of cells. They are found in various parts of the human body at every stage of development - from embryo to adult. Because stem cells are so versatile, they could potentially be used to repair and replace damaged human tissues. The purpose of experimental stem cell therapy offers potential benefits like slowing down or stopping further deterioration caused by the disease, or reversing the effects of the disease. The goal of therapy in vitiligo is to regenerate the damaged colour producing cells of the skin (melanocytes) and to correct the aberrant immune system. Normally in vitiligo the body's own immune system attacks and damages the colour producing cells of the skin, hence it is an autoimmune disease. Mesenchymal stem cells have been found to have immunomodulatory and immunosuppressive properties. Administration of mesenchymal stem cells in vitiligo prevents further damage to melanocytes and reduces disease progression. Knowledge of the melanocyte reservoir is of importance to understand the mechanism of repigmentation, which is crucial for designing newer strategies for vitiligo therapy. The possibility of skin areas without hair follicles where repigmentation occurs with a diffuse pattern suggests the existence of an amplified concept of the melanocyte reservoir that would include the intermolecular epidermis as well.

\section{Acknowledgements}

The authors are grateful to People's University, Bhopal, for providing laboratory facilities to carry out this work.

\section{Conflict of interest}

The author declares no conflict of interest.

\section{References}

1. Van Geel N, Ongenae K, Naeyaert JM. Review surgical techniques for vitiligo: a review. Dermatol. 2001;202(2):162-166.

2. Czajkowski R, Placek W, Drewa T, et al. Autologous cultured melanocytes in vitiligo treatment. Demerol Surg. 2007;33(9):1027-1036.

3. Yu H, Kumar SM, Kossenkov AV, et al. Stem cells with neural crest characteristics derived from the bulge region of cultured human hair follicles. J Invest Dermatol. 2010;130(5):1227-1236.
4. Mica Y, Lee G, Chambers SM, et al. Modeling neural crest induction, melanocyte specification, and disease-related pigmentation defects in hESCs and patient-specific iPSCs. Cell Rep. 2013;3(4):1140-1152.

5. Won Kyung S, Kyung-Mi P, Hyun-Ju K, et al. Treatment of macular degeneration using embryonic stem cell-derived retinal pigment epithelium preliminary results in Asian. patients stem cell reports. 2015;4(5):860-872.

6. Ji Young K, Dae Suk K, Hyosung S, et al. PAR-2 is involved in melanogenesis by mediating stem cell factor production in keratinocytes. Experimental Dermatol. 2016;25(6):487-489.

7. Stenn, KS, Paus R. Controls of hair follicle cycling. Physiol Rev. 2001;81(1):449-494.

8. Yoshida H, Kunisada T, Kusakabe M, et al. Distinct stages of melanocyte differentiation revealed by anlaysis of non uniform pigmentation patterns. Development. 1996;122(4):1207-1214.

9. Nowruz N, Banafshe E, Maryam Dastan I. Hair follicle stem cells: In vitro and in vivo neural differentiation. World J Stem Cells. 2015;7(5):866-872.

10. Wilson C, Cotsarelis G, Wei ZG, et al Cells within the bulge region of mouse hair follicle transiently proliferate during early anagen: heterogeneity and functional differences of various hair cycles. Differentiation. 1994;55(2):127-136.

11. Quevedo WC, Isherwoob JE. Influence of hair growth cycle on melanocyte activation in rabbit skin after a single application of methylcholanthrene. J Invest Dermatol. 1961;37:93-101.

12. Vinay K, Dogra S. Stem cells in vitiligo: Current position and prospects. World J Stem Cells. 2015;1(1):8-12.

13. Song HJ, Choi GS, Shin JH. Preservation of melanoblast of white hair follicles of segmental vitiligo lesions: A preliminary study. J Eur Acad Dermatol Venereol. 2011;25(2):240-242.

14. Nishimura EK, Jordan SA, Oshima H, et al. Dominant role of the niche in melanocyte stem-cell fate determination. Nature. 2002;416(6883):854 860 .

15. Cui J, Shen LY. Wang GC. Role of hair follicles in the repigmentation of vitiligo. J Invest Dermatol. 1991;97(3):410-416.

16. Tobin DJ, Swanson NN, Pittelkow MR, et al. Melanocytes are not absent in lesional skin of long duration vitiligo. J Pathol. 2000;191(4):407416. 